\title{
The Effects of Various Apodization Functions on the Filtering Characteristics of the Grating-Assisted SOI Strip Waveguides
}

\author{
Azadeh Karimi, Farzin Emami*, and Najmeh Nozhat \\ Optoelectronic Research Center, Faculty of Electrical Engineering, Shiraz University of Technology, \\ Shiraz, Iran
}

(Received October 23, 2013 : revised February 24, 2014 : accepted February 24, 2014)

\begin{abstract}
In this paper, four apodization functions are proposed for silicon-on-insulator (SOI) strip waveguides with sidewall-corrugated gratings. The effects of apodization functions on the full width at half maximum (FWHM), the side-lobe level, and the reflectivity of the reflection spectrum are studied using the coupled-mode theory (CMT) and the transfer-matrix method (TMM). The results show that applying proposed apodization functions creates very good filtering characteristics. Among investigated apodized waveguides, the apodization functions of Polynomial and z-power have the best performance in reducing side-lobes, where the side-lobe oscillations are entirely removed. Four functions are also used for precise adjustment of the bandwidth. Simulation results show that the minimum and maximum values of the FWHM are $0.74 \mathrm{~nm}$ and $8.48 \mathrm{~nm}$ respectively. In some investigated functions, changing the apodization parameters decreases the reflectivity which is compensated by increasing the grating length.
\end{abstract}

Keywords : Apodization, Bragg gratings, SOI strip waveguide

OCIS codes : (050.0050) Diffraction and gratings; (230.7408) Wavelength filtering devices

\section{INTRODUCTION}

Bragg gratings are used in many integrated optical components for a large variety of applications including optical signal processing, high-speed optical communications, sensing systems and networking [1-4]. Examples of prevalent grating-based components are add/drop filters for wavelength-division-multiplexing (WDM) communication systems, grating-assisted couplers, dispersion compensators, distributed-feedback and distributed-Bragg-reflector lasers [5-8].

Great compatibility with CMOS structures is made by silicon-on-insulator (SOI) technology, a promising candidate for large-scale integration of optics and electronics on a single silicon platform $[5,7,9]$. Therefore, in the past decades, SOI waveguides have been attractive choices for integration with Bragg grating structures [8, 10, 11]. A main group of SOI structures are strip waveguides which are used in many applications, including WDM add/drop filters and grating couplers $[4,12,13]$. In strip waveguides, or photonic wires, usually submicron cross sections are used $[9,10]$. Moreover, the strong confinement of light in the core due to the high index contrast between $\mathrm{Si}$ and $\mathrm{SiO}_{2}$ is a beneficial feature of strip waveguides. So, as a result of small waveguide dimensions and high confinement of light, even small perturbations on the sidewalls can result in high coupling strength [10].

Various methods are suggested for implementing Bragg gratings on the SOI waveguides. Two main categories are: (1) using ion implantation for refractive index modulation [14], and (2): physically corrugating the top surface or the sidewalls of the waveguides $[9,11,15]$.

In the grating-assisted waveguides, a part of the injected light rays reflects back around the Bragg wavelength when it propagates through the Bragg gratings. The Bragg condition is satisfied at the Bragg wavelength, $\lambda_{D}$, which is defined by [9]:

$$
\lambda_{D} \equiv 2 n_{e f f} \Lambda
$$

where $n_{\text {eff }}$ and $\Lambda$ are the effective refractive index of the fundamental mode of the waveguide and the grating

\footnotetext{
*Corresponding author: emami@sutech.ac.ir

Color versions of one or more of the figures in this paper are available online.
} 
period, respectively. However, due to the presence of some side-lobes at the vicinity of the Bragg wavelength, uniform gratings do not provide the proper performance needed in the integrated-optical components and WDM communication systems, frequently. Grating filters and multiplexers, as fundamental components in the high-speed WDM communication systems, aim to reflect a single channel in the reflection spectrum. As a result, the reduction of the side-lobes is essential for them. On the other hand, the presence of the side-lobes leads to the creation of high cross talk levels which is considered as a drawback for some applications in WDM communication systems [6]. Subsequently, the attendance of the side-lobes degrades the functionality of the communication systems. The reduction of the side-lobes is done by using the apodization concept. It is performed by gradually changing the grating strength and therefore the coupling coefficient along the waveguide [6]. By using some apodization functions, one can completely remove the side-lobe oscillations, thus resulting in smooth reflection spectra compared with reported results [9] for un-apodized waveguide. Realization of the apodization can be fulfilled by varying grating duty cycle [16] or dimensions of the waveguide [6].

In this paper, four apodization functions are introduced for SOI strip waveguides with sidewall corrugated gratings. Here, the analysis is done using the coupled-mode theory (CMT) and the transfer-matrix method (TMM). The effects of apodization functions on the side-lobe level, the full width at half maximum (FWHM), and the reflectivity of the grating reflection spectrum are investigated. Improvement of the apodization functions, in order to achieve better side-lobe reduction and filtering characteristics, is accomplished by changing the parameters of the apodization functions.

\section{THEORY}

Physical perturbation of the waveguide causes the refractive index modulation and brings the coupling between the forward and backward propagation modes. It occurs when the phase-matching condition of Eq. (1) is satisfied or simply when $\beta \equiv \pi / \Lambda$.

Consider $\Psi_{1}(x, y)$ as the transverse mode of the unperturbed waveguide that can propagate in both positive and negative directions. The electric field in the grating section can be expressed by [19]:

$$
\overrightarrow{\mathbf{E}}(x, y, z, t)=\left[F_{1}(z) \exp ^{-j \beta_{1 z}}+G_{1}(z) \exp ^{j \beta_{1 z}}\right] \Psi_{1}(x, y) \exp ^{j o t}
$$

where $\omega$ is the angular frequency of the light, $\beta$ is the propagation constant of the mode that is defined by $\beta=2 \pi n_{\text {eff }} / \lambda_{0}$, where $\lambda_{0}$ is the free-space wavelength, and $F_{1}$ and $G_{1}$ are the field amplitudes of the forward and the backward propagating modes, respectively. The electric field in Eq. (2) satisfies the wave equation [19]:

$$
\nabla^{2} \overrightarrow{\mathbf{E}}+\omega^{2} \mu \varepsilon_{0}\left[\varepsilon_{r}(x, y)+\Delta \varepsilon_{r}(x, y, z)\right] \overrightarrow{\mathbf{E}}=0
$$

where the relative permittivity, $\mathcal{E}_{r}$, is independent of the grating length while the perturbation in the relative dielectric permittivity, $\Delta \varepsilon_{r}$, is a periodic function of $z$ and can be expanded by Fourier series [19]:

$$
\Delta \varepsilon_{r}(x, y, z)=\sum_{m \neq 0} \Delta \varepsilon_{m}(x, y) \exp (-j m 2 \pi z / \Lambda)
$$

Since no gain or loss is assumed, $\varepsilon_{r}$ and $\Delta \varepsilon_{r}$ are considered to be real and so $\Delta \varepsilon_{m}=\Delta \varepsilon^{*}{ }_{-m}$, where $\Delta \varepsilon^{*}{ }_{m}$ is the complex conjugate of $\Delta \varepsilon_{m}$ [19]. Here $m=1$ is considered to obtain the coupled equations [20]. CMT can be applied for orthogonal modes [19]. The orthogonality relation is represented by the following equation [19]:

$$
\iint_{R_{2}} \Psi_{i}(x, y) \Psi_{j}(x, y) d x d y=\frac{2 \omega \mu_{0}}{\left|\beta_{i}\right|} \delta_{i j} i, j=1,2
$$

where $\mu_{0}$ is the permeability of the vacuum. By using Eq. (2)-(5) the two coupled-equations can be obtained as follows:

$$
\begin{aligned}
& \frac{d F_{1}}{d z}=-j \kappa_{11} G_{1}(z) \exp \left(j 2 \Delta \beta_{1} z\right) \\
& \frac{d G_{1}}{d z}=j \kappa_{11}{ }^{*} F_{1}(z) \exp \left(-j 2 \Delta \beta_{1} z\right)
\end{aligned}
$$

Here $\Delta \beta_{1} \equiv \beta_{1}-\pi / \Lambda$ and the coupling coefficient is defined as:

$$
\kappa_{11} \equiv \frac{\omega \varepsilon_{0}}{4} \iint_{R_{2}} \Delta \varepsilon_{1} \Psi_{1}^{2} d x d y
$$

where $\varepsilon_{0}$ is the permittivity of the vacuum and $\Delta \varepsilon_{1}$ is the first-order Fourier-expansion coefficient of the relative dielectric perturbation, $\Delta \varepsilon_{r}$. Often, grating structures can be analyzed by two methods; the direct numerical integration method and the piecewise uniform approach [21]. To solve coupled equations, as a two-point boundaryvalue problem, the shooting method and TMM can be used [21]. The shooting method requires many iterations and therefore is time consuming. However, TMM is an accurate method for analyzing grating structures [21]. In this paper, TMM, developed from the piecewise uniform approach, is used to analyze the gratings-assisted waveguides. In this method, the grating with the length of $L$ is divided into $N$ segments and the grating specifications in each segment are considered to be uniform. For each segment, 
the CMT is applied and then the transfer matrices of all segments are multiplied by each other.

Equations (6) and (7) can be rewritten in the following form [19]:

$$
\frac{d}{d z}\left(\begin{array}{l}
F_{1} \\
G_{1}
\end{array}\right)=\mathbf{S}(z)\left(\begin{array}{l}
F_{1} \\
G_{1}
\end{array}\right)
$$

where $\mathbf{S}$ is a $2 \times 2$ matrix. The relation between the fields at $z_{0}$ and $z_{1}$ can be presented as [7]:

$$
E\left(z_{0}\right)=\mathbf{C}\left(z_{0}, z_{1}\right) E\left(z_{1}\right)
$$

where $\mathbf{C}$ is the transfer matrix and $z_{0}$ and $z_{1}$ are the initial and ending positions of each segment with uniform gratings, respectively. The analytical solution of Eq. (10) can be expressed by [7]:

$$
\mathbf{C}\left(z_{0}, z_{1}\right)=\left\{\exp \left[\mathbf{S}_{1}\left(z_{1}-z_{0}\right)\right]\right\} \times\left\{\exp \left[\mathbf{S}_{2}\left(z_{1}-z_{0}\right)\right]\right\}
$$

Matrix exponentials in Eq. (11) can be solved by the Pade approximation [19] and the matrices of $\mathbf{S}_{1}$ and $\mathbf{S}_{2}$ are represented by [7]:

$$
\begin{aligned}
& \mathbf{S}_{1}=\left[\begin{array}{cc}
j \Delta \beta_{1} & 0 \\
0 & -j \Delta \beta_{1}
\end{array}\right] \\
& \mathbf{S}_{2}=\left[\begin{array}{cc}
-j \Delta \beta_{1} & -j \kappa_{11} \exp \left(2 j \Delta \beta_{1} z_{1}\right) \\
j \kappa_{11}^{*} \exp \left(-2 j \Delta \beta_{1} z_{1}\right) & j \Delta \beta_{1}
\end{array}\right]
\end{aligned}
$$

Thus, the total transfer matrix can be described as follows:

$$
\left[\begin{array}{l}
F_{1}\left(z_{0}\right) \\
G_{1}\left(z_{0}\right)
\end{array}\right]=\prod_{l=1}^{m}\left[\mathbf{C}\left(z_{l-1}, z_{l}\right)\right]\left[\begin{array}{l}
F_{1}\left(z_{m}\right) \\
G_{1}\left(z_{m}\right)
\end{array}\right]
$$

By using Eq. (14), the reflectivity and the transmittivity of the grating can be represented by:

$$
\begin{aligned}
& R=\left|\frac{G_{1}\left(z_{0}\right)}{F_{1}\left(z_{0}\right)}\right|^{2} \\
& T=\left|\frac{F_{1}\left(z_{m}\right)}{F_{1}\left(z_{0}\right)}\right|^{2}
\end{aligned}
$$

The relation between the reflectivity $R$, the coupling coefficient $\kappa_{11}$, and the grating length $L$, can be expressed as [6]:

$$
R=\tanh ^{2}\left(\kappa_{11} L\right)
$$

As it was mentioned, by using the CMT the relation between the forward and the backward propagating modes can be described through a set of equations. On the other hand, by utilizing the TMM, the grating structure is divided into $N$ segments with uniform gratings. For each segment, applying the CMT results in a transfer matrix which can relate the fields at two ends of the segment. Therefore, overall changes in the grating (along the structure as a result of the apodization functions) can be modeled by multiplying the transfer matrices of segments.

\section{SIMULATION OF SOI STRIP WAVEGUIDES WITH UNIFORM AND APODIZED GRATINGS}

Typical structures of sidewall corrugated SOI strip waveguide (SWC-SOI-SW) with uniform gratings and an exemplary structure with apodized gratings are shown in Fig. 1.

The waveguide consists of a silicon layer over the surface of a buried oxide layer with the thicknesses of 220 $\mathrm{nm}$ and $2 \mu \mathrm{m}$, respectively. For all waveguides reported in this paper, the strip width, $\mathrm{W}$, the grating period, $\Lambda$, and

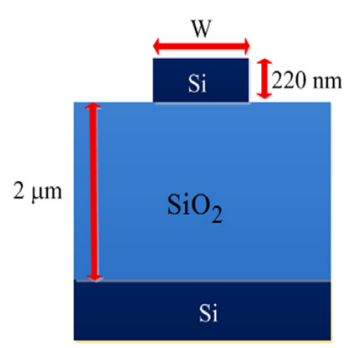

(a)

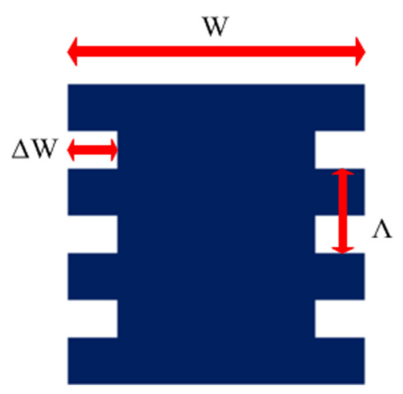

(b)

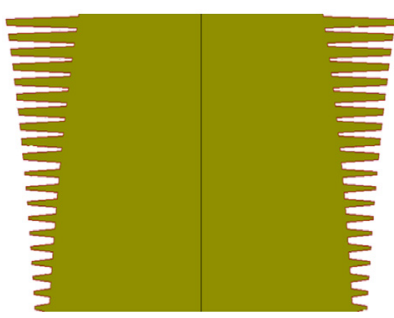

(c)

FIG. 1. (a) Cross section, (b) Top views of the SOI strip waveguide with sidewall corrugated gratings and (c) an exemplary structure for apodized gratings. 
the duty cycle are considered to be $500 \mathrm{~nm}, 310 \mathrm{~nm}$ and $50 \%$, respectively. The corrugation width, $\Delta \mathrm{W}$, and the grating length $\mathrm{L}$ are changed for different waveguides. The phase-matching condition, given in Eq. (1), and the effective refractive index of the fundamental mode of the SWC-SOI-SW are depicted in Fig. 2(a). It was assumed that the corrugation width and the grating length are $5 \mathrm{~nm}$ and $620 \mu \mathrm{m}$, respectively, The crossing point of the two diagrams in Fig. 2(a) shows the resonant wavelength of the grating structure. The transmission and the reflection spectra of the waveguide are shown in Fig. 2(b).

As is clear, the resonant peak of the transmission spectrum is compatible with the Bragg wavelength of Eq. (1). The response exhibits the FWHM of $3.31 \mathrm{~nm}$, the high side-lobe level of $-1.8 \mathrm{~dB}$, and the reflectivity of $100 \%$. Suppression of these high side-lobes can be performed by using the apodized gratings. To do this, many types of grating apodization can be utilized. The apodization functions introduced in this paper are:

Exponential 1:

$$
F\left(z^{\prime}\right)=a-b \exp \left(z^{\prime c}\right)
$$

Exponential 2:

$$
G\left(z^{\prime}\right)=\exp \left(z^{e z^{\prime}}\right)-f
$$

\section{Polynomial:}

$$
H\left(z^{\prime}\right)=j z^{\prime k}
$$

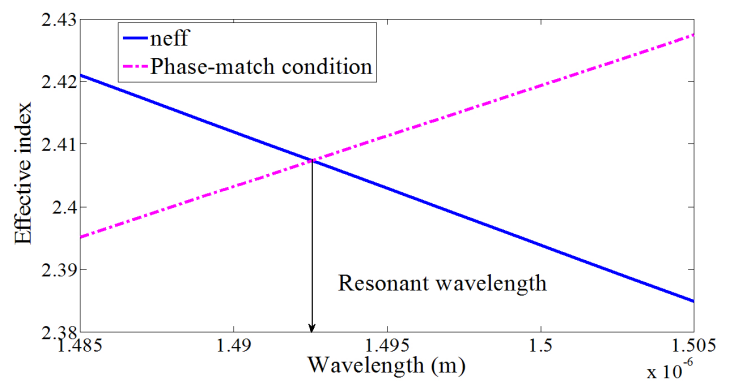

(a)

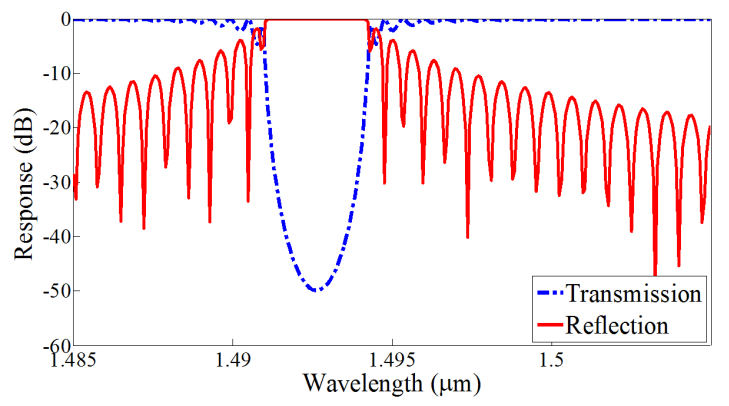

(b)

FIG. 2. (a) The effective index of the fundamental mode and the phase-matching condition versus the wavelength and (b) Transmission and reflection spectra of the SOI strip waveguide with $5 \mathrm{~nm}$ uniform gratings and the length of $620 \mu \mathrm{m}$.

$$
\begin{aligned}
& z \text {-power: } \\
& J\left(z^{\prime}\right)=m z^{\left(n z^{\prime}+p\right)}+q
\end{aligned}
$$

where $a, b, c, e, f, \mathrm{j}, k, m, n, p$ and $q$ are the apodization parameters that may be varied to optimize the filtering characteristics of the structure and $z^{\prime}=z / L$. The apodization functions are depicted in Fig. 3 .

The schematic of the apodized waveguide with the apodization function of Exponential 1, and the simulation

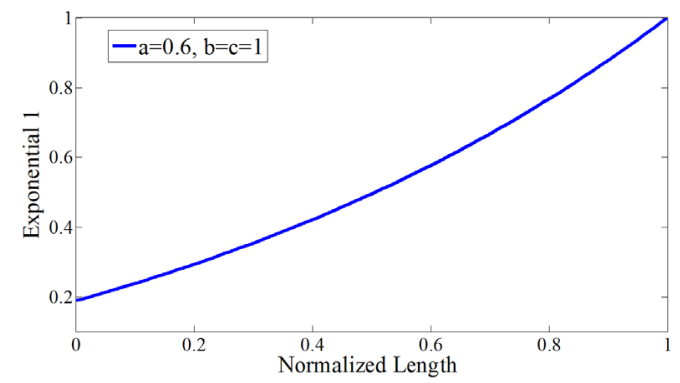

(a)

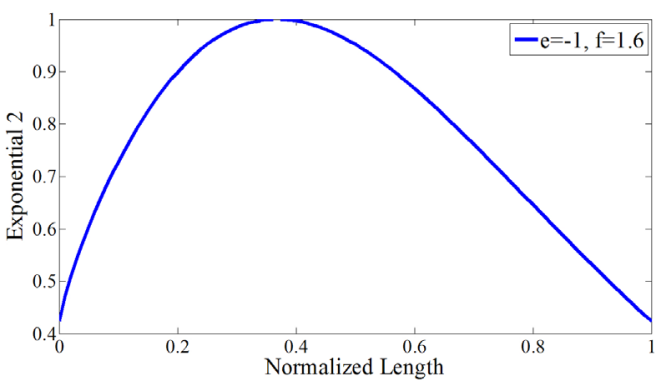

(b)

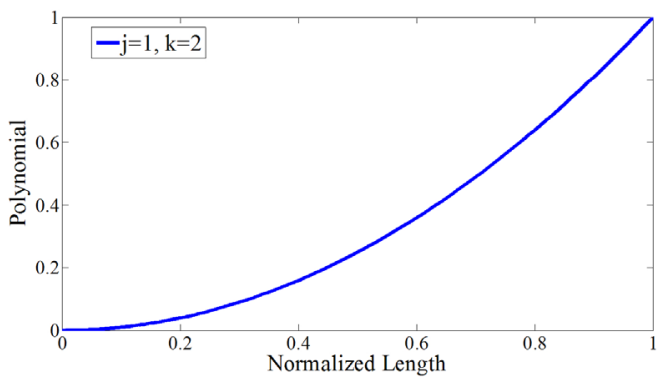

(c)

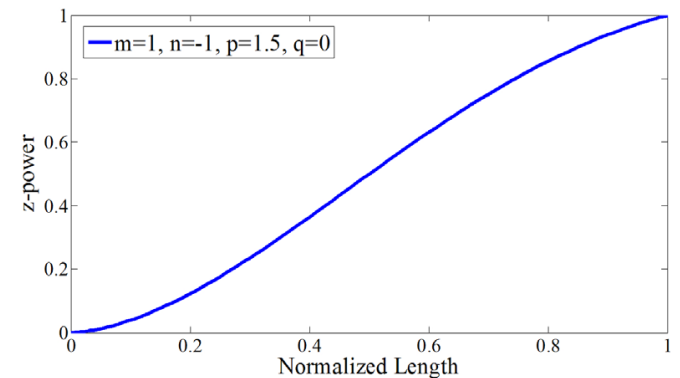

(d)

FIG. 3. Apodization functions versus the normalized length (a) Exponential 1, (b) Exponential 2, (c) Polynomial, and (d) $z$-power. 
results for different apodization parameters with the corrugation width of $5 \mathrm{~nm}$ and the grating length of $620 \mu \mathrm{m}$, are shown in Fig. 4 and summarized in Table 1.

As it is evident, by varying the apodization parameters, the value of the FWHM, the side-lobe levels and the reflectivity would be changed dramatically. An inspection

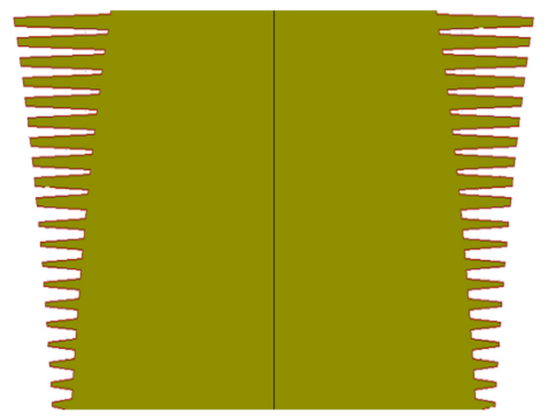

(a)

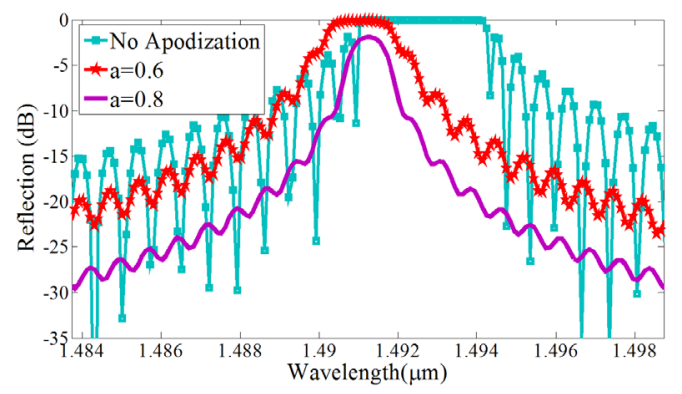

(b)

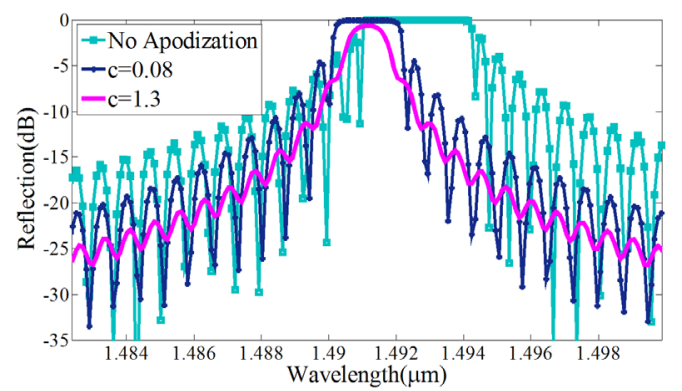

(c)

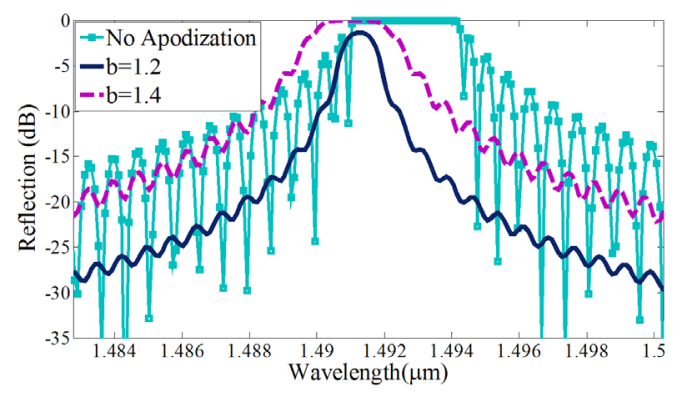

(d)

FIG. 4. (a) Schematic of the apodized waveguide with the apodization function of Exponential 1 for $a=0.6$ and $b=c=1$. Reflection spectra of the apodized waveguide for (b) $b=c=1$, $a=0.6$ and $a=0.8$, (c) $a=0.7, b=1, c=0.08$ and $c=1.3$, and (d) $a=c=1, b=1.2$ and $b=1.4$. in the results reveals that the FWHM is lower than the un-apodized or uniform waveguide. Therefore, this function can be used for applications with the requirement of the low bandwidth grating structures such as WDM filters [3, 10]. By comparing the results, it is found that, increasing the parameters $a$ and $c$ leads to a decrease in the FWHM, the side-lobe level and the reflectivity, while increasing the parameter $b$ enhances them.

On the other hand, among the reported results in Table 1, the lowest values of the FWHM and the side-lobe are $1.09 \mathrm{~nm}$ and $-15.7 \mathrm{~dB}$, respectively, which can be obtained for $b=c=1$ and $a=0.8$. However, the reflectivity is relatively low for this case. According to Eq. (17), it is possible to increase the reflectivity by increasing the grating length. The reflection spectra of the un-apodized and the apodized waveguides (with apodization function of Exponential 1 for $b=c=1, a=0.8$ and various lengths) are plotted in Fig. 5. These results are summarized in Table 2 .

As shown in Table 2, grating length increment leads to an increase in the reflectivity and the side-lobe level while the FWHM decreases and reaches below $1 \mathrm{~nm}$. Here, the minimum value of the FWHM is $0.92 \mathrm{~nm}$ which is

TABLE 1. Spectral features of the apodized waveguide with the apodization function of Exponential 1

\begin{tabular}{c|c|c|c}
\hline $\begin{array}{c}\text { Apodization } \\
\text { parameters }\end{array}$ & $\begin{array}{c}\text { FWHM } \\
(\mathrm{nm})\end{array}$ & $\begin{array}{c}\text { Side-lobe } \\
\text { level }(\mathrm{dB})\end{array}$ & $\begin{array}{c}\text { Reflectivity } \\
(\%)\end{array}$ \\
\hline No Apodization & 3.31 & -1.8 & 100 \\
\hline$b=c=1, a=0.6$ & 1.96 & -8.14 & 98 \\
\hline$b=c=1, a=0.7$ & 1.46 & -11.32 & 90 \\
\hline$b=c=1, a=0.8$ & 1.09 & -15.7 & 80 \\
\hline$a=c=1, b=1.2$ & 1.19 & -14.22 & 74 \\
\hline$a=c=1, b=1.3$ & 1.76 & -9.45 & 96 \\
\hline$a=c=1, b=1.4$ & 3.02 & -5.8 & 99 \\
\hline$a=0.7, b=1, c=0.08$ & 2.09 & -4.6 & 99 \\
\hline$a=0.7, b=1, c=0.3$ & 1.81 & -6.1 & 98 \\
\hline$a=0.7, b=1, c=1.3$ & 1.36 & -11.26 & 87 \\
\hline
\end{tabular}

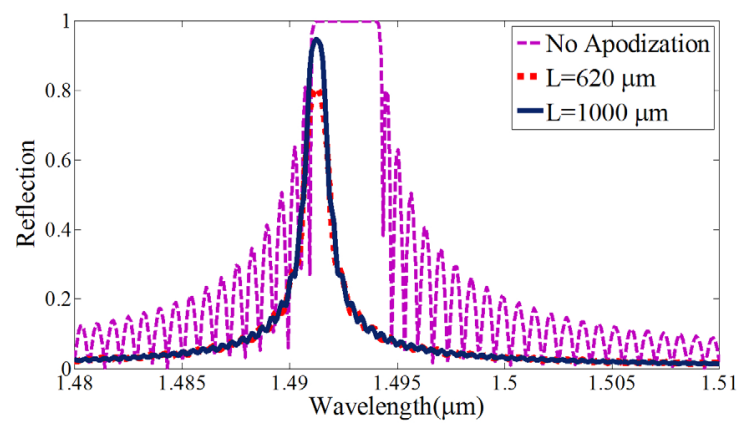

FIG. 5. Comparison between the reflection spectra of the un-apodized and the apodized waveguides with the apodization function of Exponential 1 for $b=c=1, a=0.8$ and different grating lengths. 
TABLE 2. Spectral features of the apodized waveguide with the apodization function of Exponential 1 for $b=c=1, a=0.8$ and different lengths

\begin{tabular}{c|c|c|c}
\hline \hline $\begin{array}{c}\text { Grating Length }(L) \\
(\mu \mathrm{m})\end{array}$ & $\begin{array}{c}\text { FWHM } \\
(\mathrm{nm})\end{array}$ & $\begin{array}{c}\text { Side-lobe } \\
\text { level }(\mathrm{dB})\end{array}$ & $\begin{array}{c}\text { Reflectivity } \\
(\%)\end{array}$ \\
\hline$L=620$ & 1.09 & -15.7 & 80 \\
\hline$L=820$ & 0.97 & -13.1 & 85 \\
\hline$L=1000$ & 0.92 & -11.4 & 94 \\
\hline
\end{tabular}

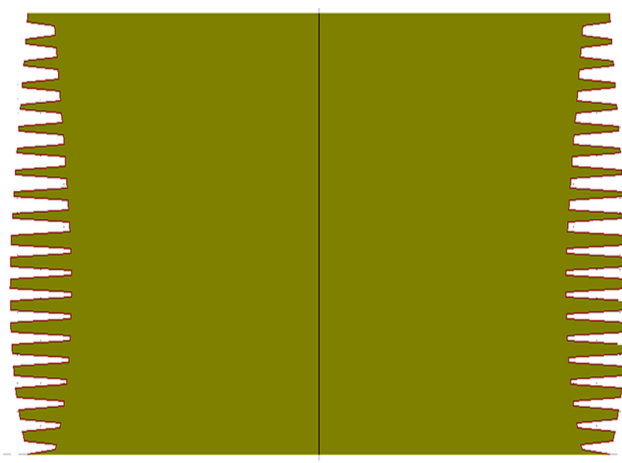

(a)

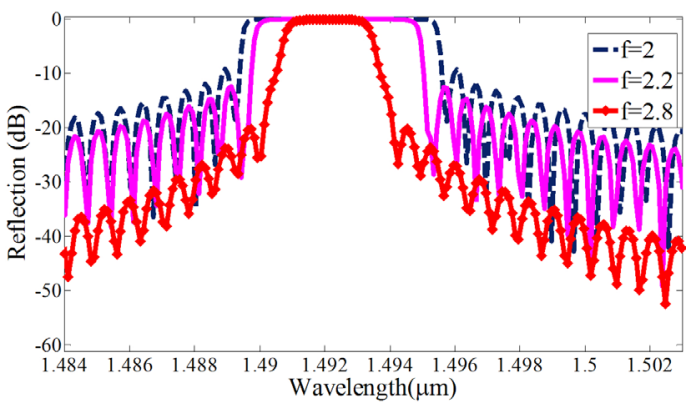

(b)

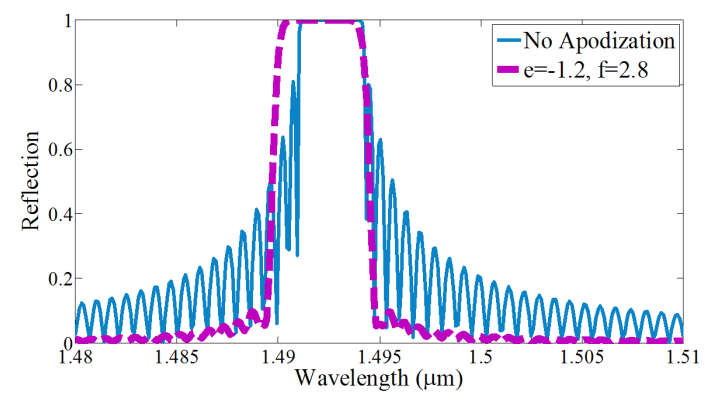

(c)

FIG. 6. (a) Schematic of the apodized waveguide with the apodization function of Exponential 2 for $e=-1, f=1.6$, (b) Reflection spectra of the apodized waveguide for: $e=-1, f=2$, $f=2.2$ and $f=2.8$, and (c) Comparison between the reflection spectra of the apodized and un-apodized waveguides with 2 $\mathrm{nm}$ and $5 \mathrm{~nm}$ gratings, respectively.

obtained for $L=1000 \mu \mathrm{m}$.

In Fig. 6 the schematic and the spectra of the apodized waveguide with the apodization function of Exponential 2 are shown and the simulation results are presented in Table 3 in summary.

The simulations of apodized waveguides are performed using the structures with the grating length of $620 \mu \mathrm{m}$ and the corrugation width of $2 \mathrm{~nm}$. As it is clear, compared to the un-apodized waveguide with the corrugation width of $5 \mathrm{~nm}$, in some cases, the FWHM of the apodized waveguide is increased considerably. From Table 3, it can be seen that by enhancing the apodization $f$-parameter, the FWHM decreases dramatically. Moreover, the side-lobe level decreases when this parameter increases from $f=1.6$ to $f=2.8$ and then increases for the higher values. From Table 3, it can be seen that the high and low values of the FWHM with the desired values of the side-lobe level and the reflectivity can be attained by adjusting the apodization parameters. Among these functions, the maximum FWHM of $8.48 \mathrm{~nm}$ is obtained by applying the apodization function of Exponential 2 for $e=-1$ and $f=1.6$.

The schematic of the apodized waveguide with the apodization function of Polynomial is plotted in Fig. 7(a). The simulation results for the grating length of $620 \mu \mathrm{m}$ and the corrugation width of $5 \mathrm{~nm}$ are shown in Figs. 7(b), (c). These results are summarized in Table 4.

As shown in Fig. 7, the apodized waveguide presents extremely good filtering behavior and the side-lobe oscillations of the reflection spectra are entirely removed. The calculated smooth spectra can be utilized in applications such as WDM communication systems where the presence of side-lobes is considered to be a drawback. Fig. 7(b) demonstrates the impact of changing the apodization $k$-parameter on the amplitude of the side-lobes, for apodization function of Polynomial type. As this parameter increases, the amplitudes of the side-lobes gradually

TABLE 3. Spectral features of the apodized waveguide with the apodization function of Exponential 2

\begin{tabular}{c|c|c|c}
\hline $\begin{array}{c}\text { Apodization } \\
\text { parameters }\end{array}$ & $\begin{array}{c}\text { FWHM } \\
(\mathrm{nm})\end{array}$ & $\begin{array}{c}\text { Side-lobe } \\
\text { level }(\mathrm{dB})\end{array}$ & $\begin{array}{c}\text { Reflectivity } \\
(\%)\end{array}$ \\
\hline No Apodization & 3.31 & -1.8 & 100 \\
\hline$e=-1, f=1.6$ & 8.48 & -3 & 99 \\
\hline$e=-1, f=1.8$ & 7.04 & -6.2 & 99 \\
\hline$e=-1, f=2$ & 6.00 & -9.2 & 99 \\
\hline$e=-1, f=2.2$ & 5.1 & -12.5 & 99 \\
\hline$e=-1, f=2.4$ & 4.18 & -16.2 & 99 \\
\hline$e=-1, f=2.6$ & 3.37 & -20 & 99 \\
\hline$e=-1, f=2.8$ & 2.67 & -20.18 & 99 \\
\hline$e=-1, f=3$ & 2.19 & -14 & 93 \\
\hline$e=-1, f=3.2$ & 1.96 & -1.5 & 70 \\
\hline$e=-0.8, f=2.8$ & 1.618 & -17.2 & 85 \\
\hline$e=-0.7, f=2.8$ & 1.29 & -19.8 & 63 \\
\hline$e=-1.1, f=2.8$ & 3.46 & -20.3 & 99 \\
\hline$e=-1.2, f=2.8$ & 4.55 & -20.13 & 99 \\
\hline & & &
\end{tabular}


diminish and reach to zero for $k=2$, and the reflectivity is $96 \%$. By further increasing $k$, the spectrum remains smooth, but the reflectivity is reduced. As shown in Fig. 7 (c), by choosing $k=2$ and changing the $j$-parameter, the FWHM of the spectra can be adjusted. For low values of $j$, the FWHM becomes narrower than that of the un-apodized waveguide while the higher values of $j$ lead to wider spectra compared to the un-apodized one. Based on the results listed in Table 4 , it can be seen that the minimum FWHM of $1.24 \mathrm{~nm}$ is achieved for the parameters $k=2$ and $j=0.6$ with the completely smooth spectrum. However, in this case, the reflectivity is small. It can be increased by increasing the grating length.

The impacts of enhancing the grating length on the reflection spectra of the apodized structure with the apodization function of Polynomial are shown in Fig. 8 and brief results are given in Table 5 .

Comparing the simulation results of un-apodized structure,

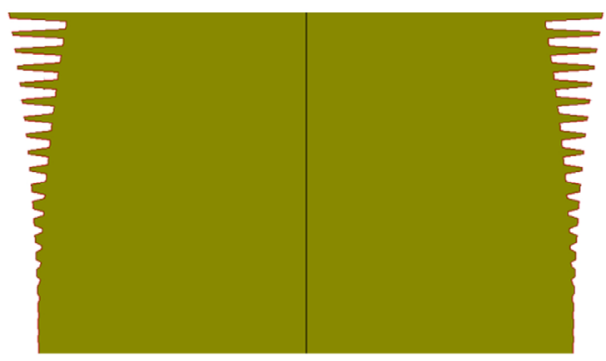

(a)

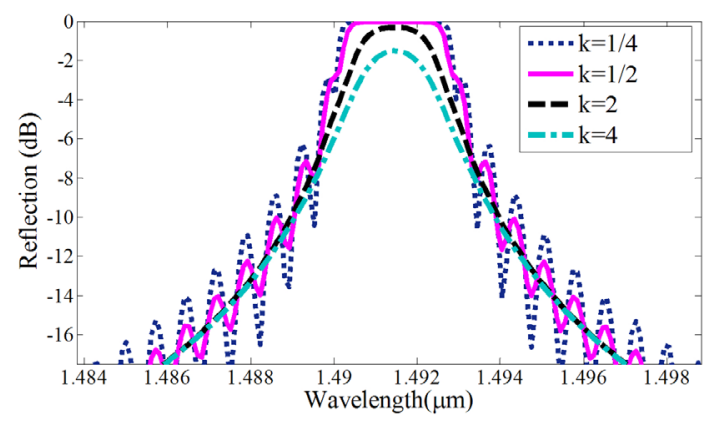

(b)

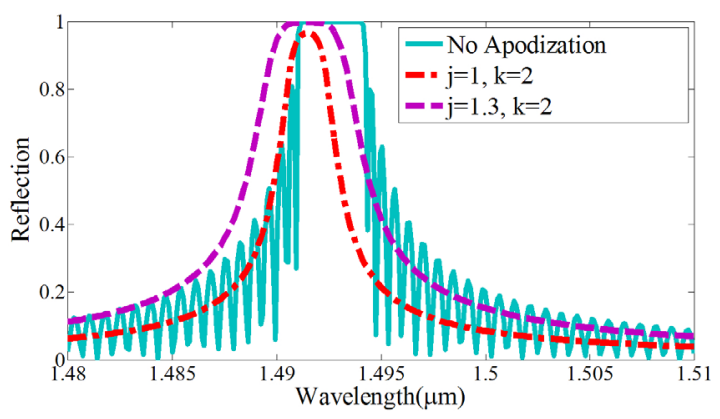

(c)

FIG. 7. (a) Schematic of the apodized waveguide with the apodization function of Polynomial for $j=1, k=2$. Reflection spectra of the apodized waveguide for: (b) $j=1, k=1 / 4, k=1 / 2$, $k=2$ and $k=4$, (c) $k=2, j=1$ and $j=1.3$. it is clear that the completely-flat apodized spectra are much narrower than that of the un-apodized one. For $L=1550 \mu \mathrm{m}$, the reflectivity reaches above $90 \%$ and the FWHM becomes $0.87 \mathrm{~nm}$, that is too small in comparison with the reported results of the un-apodized waveguide [9].

The schematic of the apodized waveguide with the last apodization function of z-power is shown in Fig. 9(a). Moreover, the simulation results of applying the apodization on the waveguide with the length of $620 \mu \mathrm{m}$ and the corrugation width of $5 \mathrm{~nm}$ are presented in Fig. 9(b) and 9(c), with a summarization reported in Table 6 .

As shown in Fig. 9(b), by increasing the apodization

TABLE 4. Spectral features of the apodized waveguide with the apodization function of Polynomial

\begin{tabular}{c|c|c|c}
\hline $\begin{array}{c}\text { Apodization } \\
\text { parameters }\end{array}$ & $\begin{array}{c}\text { FWHM } \\
(\mathrm{nm})\end{array}$ & $\begin{array}{c}\text { Side-lobe } \\
\text { level }(\mathrm{dB})\end{array}$ & $\begin{array}{c}\text { Reflectivity } \\
(\%)\end{array}$ \\
\hline No Apodization & 3.31 & -1.8 & 100 \\
\hline$j=1, k=1 / 4$ & 2.85 & -2.89 & 99 \\
\hline$j=1, k=1 / 2$ & 3.03 & -7.2 & 99 \\
\hline$j=1, \quad k=2$ & 2.4 & 0 & 96 \\
\hline$j=1, \quad k=4$ & 2.34 & 0 & 70 \\
\hline$j=0.6, \quad k=2$ & 1.24 & 0 & 60 \\
\hline$j=0.7, \quad k=2$ & 1.4 & 0 & 71 \\
\hline$j=0.9, \quad k=2$ & 1.96 & 0 & 86 \\
\hline$j=1.1, \quad k=2$ & 3.1 & 0 & 97 \\
\hline$j=1.3, \quad k=2$ & 4.67 & 0 & 99 \\
\hline
\end{tabular}

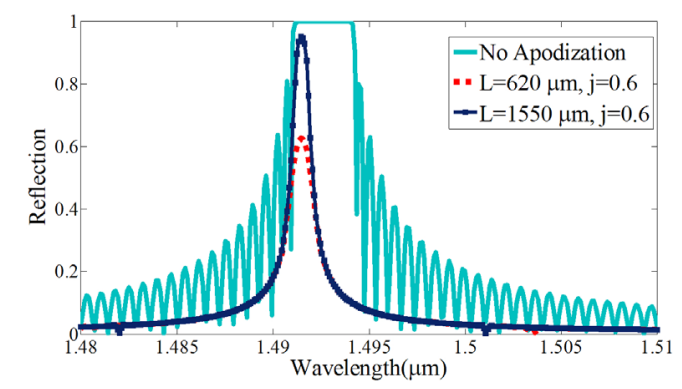

FIG. 8. Comparison between the reflection spectra of the un-apodized and the apodized waveguides with the apodization function of Polynomial for $k=2, j=0.6$ and different lengths.

TABLE 5. Spectral features of the apodized waveguide with the apodization function of Polynomial for $j=0.6, k=2$ and different lengths

\begin{tabular}{c|c|c|c}
\hline \hline $\begin{array}{c}\text { Grating Length (L) } \\
(\mu \mathrm{m})\end{array}$ & $\begin{array}{c}\text { FWHM } \\
(\mathrm{nm})\end{array}$ & $\begin{array}{c}\text { Side-lobe } \\
\text { level }(\mathrm{dB})\end{array}$ & $\begin{array}{c}\text { Reflectivity } \\
(\%)\end{array}$ \\
\hline 620 & 1.24 & 0 & 60 \\
\hline 1000 & 0.96 & 0 & 69 \\
\hline 1550 & 0.87 & 0 & 95 \\
\hline
\end{tabular}


p-parameter, the side-lobe oscillations gradually diminish until they are totally removed for $p=1.5$. By further increasing of this parameter, the spectrum remains flat, but the FWHM and the reflectivity are reduced.

A comparison between the un-apodized and apodized waveguides for $m=1, n=-1, p=3$ and $q=0$ is illustrated in Fig. 9(c). As it is clear, utilizing the apodization has considerable impact on improving the filtering characteristics of the structure and a totally-flat reflection spectrum can be obtained. Absence of the side-lobes enhances the performance of the structure and makes it an attractive choice to be used in applications such as WDM communication systems, where high level side-lobes lead to cross talk between adjacent channels.

On the other hand, by decreasing the apodization $q$ - and $n$-parameters, the FWHM, the side-lobe level and the reflectivity would be decreased. Based on the results of

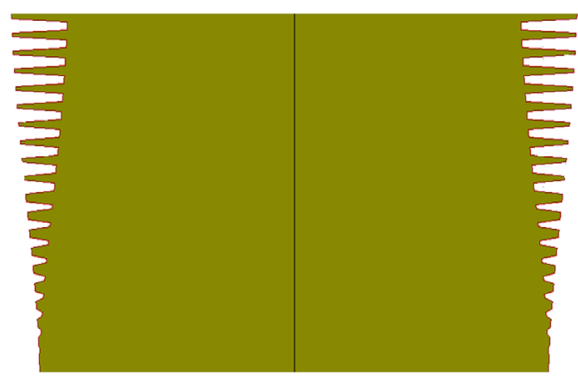

(a)

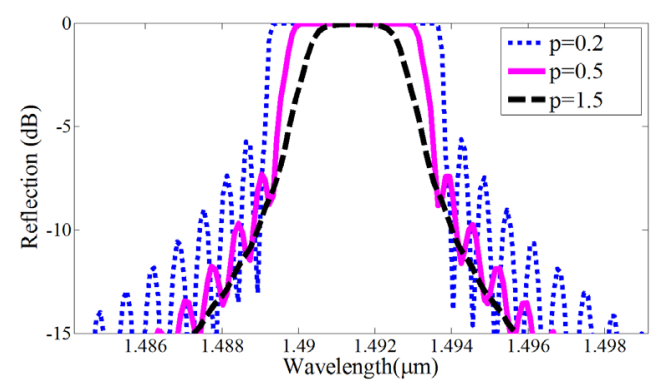

(b)

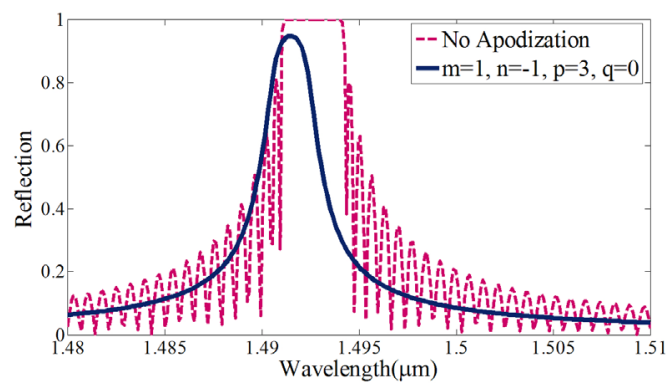

(c)

FIG. 9. (a) Schematic of the apodized waveguide with the apodization function of $z$-power for $m=1, n=-1, p=1.5, q=0$. Reflection spectra of the apodized waveguide for: (b) $m=1$, $n=-1, q=0, p=0.2, p=0.5$ and $p=1.5$ and (c) Comparison between the un-apodized and the apodized waveguides for $m=1, n=-1, p=3$ and $q=0$.
Table 6, the minimum value of the FWHM is $1.05 \mathrm{~nm}$ for $m=1, n=-1, p=0$ and $q=-1$. However, in this case the reflectivity is small. Figure 10 shows the spectra of the apodized waveguide for $m=1, n=-1, p=0, q=-1$, with different lengths. Also, the results of length increment are listed in Table 7.

As can be deduced from Fig. 10 and Table 7, higher reflectivity and lower FWHM are obtained by increasing the grating length. By comparing the spectra of un-apodized and the apodized waveguides, it is found that much narrower spectra with lower side-lobes can be achieved by

TABLE 6. Spectral features of the apodized waveguide with the apodization function of $z$-power

\begin{tabular}{c|c|c|c}
\hline \hline $\begin{array}{c}\text { Apodization } \\
\text { parameters }\end{array}$ & $\begin{array}{c}\text { FWHM } \\
(\mathrm{nm})\end{array}$ & $\begin{array}{c}\text { Side-lobe } \\
\text { level }(\mathrm{dB})\end{array}$ & $\begin{array}{c}\text { Reflectivity } \\
(\%)\end{array}$ \\
\hline No Apodization & 3.31 & -1.8 & 100 \\
\hline$m=1, n=-1, p=0, q=-0.5$ & 2.76 & -6 & 99 \\
\hline$m=1, n=-1, p=0, q=-0.7$ & 1.88 & -9.9 & 98 \\
\hline$m=1, n=-1, p=0, q=-1$ & 1.05 & -22.2 & 65 \\
\hline$m=-1, n=7, p=0, q=0.8$ & 1.88 & -9.2 & 97 \\
\hline$m=-1, n=5, p=0, q=0.8$ & 1.59 & -15.9 & 89 \\
\hline$m=-1, n=3, p=0, q=0.8$ & 1.2 & -17.5 & 49 \\
\hline$m=1, n=-1, p=0.2, q=0$ & 4.6 & -5.7 & 100 \\
\hline$m=1, n=-1, p=0.5, q=0$ & 3.77 & -7.3 & 99 \\
\hline$m=1, n=-1, p=1.5, q=0$ & 2.9 & 0 & 99 \\
\hline$m=1, n=-1, p=3, q=0$ & 2.49 & 0 & 94 \\
\hline
\end{tabular}

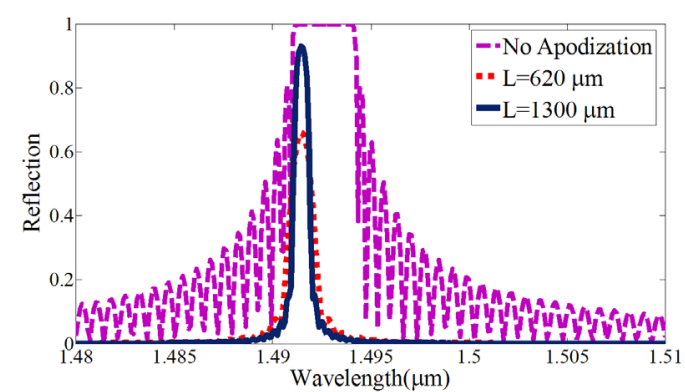

FIG. 10. Comparison between the reflection spectra of the un-apodized and the apodized waveguides with the apodization function of $z$-power for $m=1, n=-1, p=0, q=-1$ and different lengths.

TABLE 7. Spectral features of the apodized waveguide with the apodization function of $z$-power for $m=1, n=-1, p=0$, $q=-1$ and different lengths

\begin{tabular}{c|c|c|c}
\hline $\begin{array}{c}\text { Grating Length }(\mathrm{L}) \\
(\mu \mathrm{m})\end{array}$ & $\begin{array}{c}\text { FWHM } \\
(\mathrm{nm})\end{array}$ & $\begin{array}{c}\text { Side-lobe } \\
\text { level }(\mathrm{dB})\end{array}$ & $\begin{array}{c}\text { Reflectivity } \\
(\%)\end{array}$ \\
\hline 620 & 1.05 & -22.2 & 65 \\
\hline 1000 & 0.81 & -18.36 & 86 \\
\hline 1300 & 0.74 & -23.1 & 93 \\
\hline
\end{tabular}


using the apodization. Moreover, the FWHM of $0.74 \mathrm{~nm}$ for the grating length of $1300 \mu \mathrm{m}$ is the minimum value among the apodized waveguides proposed in this paper.

\section{CONCLUSIONS}

In this paper, four apodization functions, applied to SOI strip waveguide with sidewall corrugated gratings are proposed. The effects of apodization functions on the FWHM, the side-lobe level, and the reflectivity are studied. Compared to the reported results for un-apodized waveguide by Wang et al. [9], proposed apodization functions bring considerable enhancement in filtering behavior of the structure. To reduce the side-lobes, the best performed apodization functions are Polynomial and z-power, which result in the completely smooth reflection spectra, without any side-lobe oscillations. By applying the apodization of Exponential 1 and Exponential 2 on the $620-\mu \mathrm{m}$-long waveguides, the low-value side-lobes of $-15.7 \mathrm{~dB}$ with too small fluctuations and $-20.18 \mathrm{~dB}$ are obtained, respectively. In addition to removing the oscillations and reducing the side-lobe level, four functions are also used to adjust the bandwidth. The apodization functions of Exponential 1, Polynomial and z-power, substantially decrease the FWHM while Exponential 2 can be applied to increase the FWHM. By comparing the results, it is clear that the minimum value of the FWHM is $0.74 \mathrm{~nm}$ that is obtained for the $1300-\mu \mathrm{m}$-long waveguide with the apodization function of $z$-power. On the other hand, using the apodization function of Exponential 2, leads to the maximum value of the FWHM of $8.48 \mathrm{~nm}$, for the $620-\mu \mathrm{m}-$ long waveguide. In order to increase the reflectivity of the apodized waveguides with different functions, the simulations are also performed for various grating lengths.

\section{REFERENCES}

1. P. Prabhathan, V. Murukeshan, Z. Jing, and P. V. Ramana, "Compact SOI nanowire refractive index sensor using phase shifted Bragg grating," Opt. Express 17, 15330-15341 (2009).

2. L. Vivien, D. Pascal, S. Lardenois, D. Marris-Morini, E. Cassan, F. Grillot, S. Laval, J.-M. Fédéli, and L. El Melhaoui, "Light injection in SOI microwaveguides using high-efficiency grating couplers," J. Lightwave Technol. 24, 3810-3815 (2006)

3. O. Ozolinšs and Ģ. Ivanovs, "Realization of optimal FBG band-pass filters for high speed HDWDM," Lithuanian J. Electron. Elec. Eng. 4, 41-44 (2009).

4. C. Riziotis and M. N. Zervas, "Novel full-cycle-couplerbased optical add-drop multiplexer and performance characteristics at 40-Gb/s WDM networks," J. Lightwave Technol. 21, 1828-1837 (2003).

5. W. Shi, X. Wang, W. Zhang, L. Chrostowski, and N. A. F. Jaeger, "Contradirectional couplers in silicon-on-insulator rib waveguides," Opt. Lett. 36, 3999-4001 (2011).

6. J. T. Hastings, M. H. Lim, J. G. Goodberlet, and H. I.
Smith, "Optical waveguides with apodized sidewall gratings via spatial-phase-locked electron-beam lithography," J. Vac. Sci. Technol. B 20, 2753-2757 (2002).

7. W. Shi, X. Wang, C. Lin, H. Yun, Y. Liu, T. Baehr-Jones, M. Hochberg, N. A. F. Jaeger, and L. Chrostowski, "Silicon photonic grating-assisted, contra-directional couplers," Opt. Express 21, 3633-3650 (2013).

8. T. E. Murphy, J. T. Hastings, and H. I. Smith, "Fabrication and characterization of narrow-band Bragg-reflection filters in silicon-on-insulator ridge waveguides," J. Lightwave Technol. 19, 1938-1942 (2001).

9. X. Wang, W. Shi, R. Vafaei, N. A. F. Jaeger, and L. Chrostowski, "Uniform and sampled Bragg gratings in SOI strip waveguides with sidewall corrugations," IEEE Photon. Technol. Lett. 23, 290-292 (2011).

10. X. Wang, W. Shi, H. Yun, S. Grist, N. A. F. Jaeger, and L. Chrostowski, "Narrow-band waveguide Bragg gratings on SOI wafers with CMOS-compatible fabrication process," Opt. Express 20, 15547-15558 (2012).

11. X. Wang, W. Shi, R. Vafaei, N. A. F Jaeger, and L. Chrostowski, "Silicon-on-insulator Bragg gratings fabricated by deep UV lithography," in Proc. ACP (Shanghai, China, Dec. 2010), pp. 501-502.

12. D. T. H. Tan, K. Ikeda, S. Zamek, A. Mizrahi, M. P. Nezhad, A. V. Krishnamoorthy, K. Raj, J. E. Cunningham, X. Zheng, I. Shubin, Y. Luo, and Y. Fainman, "Wide bandwidth, low loss 1 by 4 wavelength division multiplexer on silicon for optical interconnects," Opt. Express 19, 2401-2409 (2011).

13. D. Taillaert, P. Bienstman, and R. Baets, "Compact efficient broadband grating coupler for silicon-on-insulator waveguides," Opt. Lett. 29, 2749-2751 (2004).

14. M. P. Bulk, A. P. Knights, P. E. Jessop, P. Waugh, R. Loiacono, G. Z. Mashanovich, G. T. Reed, and R. M. Gwilliam, "Optical filters utilizing ion implanted Bragg gratings in SOI waveguides," Adv. Opt. Technol. 1, 1-6 (2008).

15. S. Honda, Z. Wu, J. Matsui, K. Utaka, T. Edura, M. Tokuda, K. Tsutsui, and Y. Wada, "Largely-tunable wideband Bragg gratings fabricated on SOI rib waveguides employed by deep-RIE," Electron. Lett. 43, 630-631 (2007).

16. D. Wiesmann, C. David, R. Germann, D. Emi, and G. Bona, "Apodized surface-corrugated gratings with varying duty cycles," IEEE Photon. Technol. Lett. 12, 639-641 (2000).

17. J. He, X. Sun, and M. Zhang, "A novel add/drop multiplexer architecture for DWDM network," J. Infrared and Millimeter Waves 21, 57-62 (2000).

18. P. De Heyn, S. Verstuyft, S. Keyvaninia, A. Trita, and D. Van Thourhout, "Tunable 4-channel ultra-dense WDM demultiplexer with III-V photodiodes integrated on silicon-oninsulator," in Proc. ACP (Guangzhou, China, Nov. 2012), pp. 1-3, ATh2B.

19. J. P. Weber, "Spectral characteristics of coupled-waveguide Bragg-reflection tunable optical filter," Optoelectronics, IEE Proceedings J. 140, 275-284 (1993).

20. Ch. Riziotis and M. N. Zervas, "Design considerations in optical add/drop multiplexers based on grating-assisted null couplers," J. Lightwave Technol. 19, 92-104 (2001).

21. G. J. Liu, Q. Li, G. L. Jin, and B. M. Liang, "Transfer matrix method analysis of apodized grating couplers," Opt. Commun. 235, 319-324 (2004). 